\title{
Aero-engine Fault Diagnosis Using a Feature Weighting Fuzzy Clustering Algorithm
}

\author{
Yuan Zhou ${ }^{1, *}$, Hong-Fu Zuo ${ }^{2}$ and Jun $\mathrm{He}^{3}$ \\ ${ }^{1,3}$ College of Electronic and Information Engineering, Nanjing University of \\ Information Science and Technology, 219 Ningliu Road, Nanjing, 210044, China \\ ${ }^{1,2}$ College of Civil Aviation, Nanjing University of Aeronautics and Astronautics, \\ 29 Yudao Street, Nanjing 210016, China \\ ${ }^{1}$ zhouyuan@nuist.edu.cn
}

\begin{abstract}
Aero-engine fault diagnosis based on multi-sensor data is still a challenge for lacking of fault samples and high complexity of data. This paper proposed a new aero-engine fault diagnosis method by using a feature weighting fuzzy compactness and separation clustering algorithm (WFCS). WFCS can handle high-dimensional data well by considering the feature weighting effect on clustering. Furthermore, WFCS can cluster the aero-engine multi-sensor data reasonably by getting the maximum separation of between-cluster and compactness of with-in clusters. The proposed method was validated both on simulated dataset and real dataset and had the best average results, which demonstrates the efficacy of the proposed approach.
\end{abstract}

Keywords: aero-engine fault diagnosis; fuzzy clustering; fuzzy compactness and separation; feature weighting

\section{Introduction}

Aero-engine fault diagnosis is a very important task in aero-engine health management, which can facilitate crucial decision making [1-4]. Effective fault diagnosis can reduce the cost and complexity of the maintenance procedure and improve the reliability etc. The multi-sensor data can be used to interpret which part of an aero-engine is degrading or has fault. Fault diagnosis based on multi-sensor data is still an intricate problem and remains as a major challenge to be applied on complex engineered systems [5]. Also, it is still a challenge for fault diagnosis approaches to handle small sample size, high dimensional multi-sensor data and classify these data effectively.

Clustering is a pattern recognition method and can classify data into different clusters, which the same clusters are similar to each other and data from different clusters are dissimilar [6-7]. After Zadeh introduced the fuzzy set concept in 1965[8], the fuzzy Cmeans (FCM) clustering algorithm [9-11] becomes a classical unsupervised pattern recognition method. Huang et.al., improved k-means clustering algorithm with automated variable weighting [12] and Wang et. al., extended FCM with variable weighting [13]. These feature weighting clustering algorithms based on FCM cluster data only according to the distances between data points and cluster centroids. Fuzzy compactness and separation algorithm(FCS) proposed by $\mathrm{Wu}$ et al. [14] try to get the maximum separation of between-cluster and compactness of with-in clusters, which is more reasonable than FCM.

In this paper, we propose a new aero-engine fault diagnosis method using a feature weighting fuzzy compactness and separation clustering algorithm (WFCS). This paper is organized as follows. Section 2 introduces the FCS, WFCS algorithms and discusses the adjustment of WFCS. Section 3 conducts extensive experiments on both simulated data 
and real data, and compares WFCS with FCS and WFCM. Section 4 concludes this paper and points out the future work.

\section{Related Work}

\subsection{FCS Algorithm}

$X=\left\{x_{1}, x_{2}, \ldots, x_{n}\right\}$ is a dataset in an $s$-dimensional space $\mathrm{R}^{s}$ and $c$ is the number of clusters. $a_{1}, \ldots, a_{c}$ is a mutually disjoint cluster centroids sets and $a_{i} \in \mathrm{R}^{s} . \bar{X}=\frac{1}{n} \sum_{j=1}^{n} x_{j}$ denotes the grand mean of $X$.

Objective function is defined as:

$$
J_{F C S}=S_{F W}-\eta S_{F B}=\sum_{i=1}^{c} \sum_{j=1}^{n} \mu_{i j}^{m}\left\|x_{j}-a_{i}\right\|^{2}-\sum_{i=1}^{c} \eta_{i} \sum_{j=1}^{n} \mu_{i j}^{m}\left\|a_{i}-\bar{X}\right\|^{2}
$$

where the membership value of the $j$ th data point to the $i$ th cluster is $\mu_{i j} \in[0,1]$, and fuzzy exponent $m>1$.

By minimizing $J_{F C S}$, it produces $c$ clusters by update equations as Equation (2) and Equation (3):

$$
\begin{gathered}
a_{i}=\frac{\sum_{j=1}^{n} \mu_{i j}^{m} x_{j}-\eta_{i} \sum_{j=1}^{n} \mu_{i j}^{m} \bar{X}}{\sum_{j=1}^{n} \mu_{i j}^{m}-\eta_{i} \sum_{j=1}^{n} \mu_{i j}^{m}} \\
\mu_{i j}=\frac{\left(\left\|x_{j}-a_{i}\right\|^{2}-\eta_{i}\left\|a_{i}-\bar{X}\right\|^{2}\right)^{\frac{-1}{m-1}}}{\sum_{t=1}^{c}\left(\left\|x_{j}-a_{t}\right\|^{2}-\eta_{t}\left\|a_{t}-\bar{X}\right\|^{2}\right)^{\frac{-1}{m-1}}}
\end{gathered}
$$

The parameter $\eta_{i}$ can be set up with Equation (4).

$$
\eta_{i}=\frac{\beta}{4} \frac{\min _{i \neq i^{\prime}}\left\|a_{i}-a_{i^{\prime}}\right\|^{2}}{\max _{t}\left\|a_{t}-\bar{X}\right\|^{2}}
$$

where $0 \leq \beta \leq 1$ and $t=1, \ldots, c$.

\subsection{WFCS Algorithm}

First, we define the objective function as follows:

$$
J_{W F C S}=\sum_{i=1}^{c} \sum_{j=1}^{n} \sum_{k=1}^{s} \mu_{i j}^{m} \omega_{k}^{\alpha}\left\|x_{j k}-a_{i k}\right\|^{2}-\sum_{i=1}^{c} \sum_{j=1}^{n} \sum_{k=1}^{s} \eta_{i} \mu_{i j}^{m} \omega_{k}^{\alpha}\left\|a_{i k}-\overline{X_{k}}\right\|^{2}
$$

WFCS can be presented as the following optimization problem:

$$
\left\{\begin{array}{l}
\min J_{W F C S} \\
\text { s.t. } \sum_{i=1}^{c} \mu_{i j}=1 \quad, \sum_{k=1}^{s} \omega_{k}=1
\end{array}\right.
$$

By using the Lagrange multiplier to solve Equation (6), we have the following update equations: 


$$
\begin{gathered}
a_{i k}=\frac{\sum_{j=1}^{n} \mu_{i j}^{m}\left(x_{j k}-\eta_{i} \overline{X_{k}}\right)}{\sum_{j=1}^{n} \mu_{i j}^{m}\left(1-\eta_{i}\right)} \\
\omega_{k}=\frac{\left(\sum_{i=1}^{c} \sum_{j=1}^{n} \mu_{i j}^{m}\left(\left\|x_{j k}-a_{i k}\right\|^{2}-\eta_{i}\left\|a_{i k}-\overline{X_{k}}\right\|^{2}\right)\right)^{\frac{1}{1-\alpha}}}{\sum_{t=1}^{s}\left(\sum_{i=1}^{c} \sum_{j=1}^{n} \mu_{i j}^{m}\left(\left\|x_{j t}-a_{i t}\right\|^{2}-\eta_{i}\left\|a_{i t}-\overline{X_{t}}\right\|^{2}\right)\right)^{\frac{1}{1-\alpha}}} \\
\mu_{i j}=\frac{\left(\sum_{k=1}^{s} \omega_{k}^{\alpha}\left(\left\|x_{j k}-a_{i k}\right\|^{2}-\eta_{i}\left\|a_{i k}-\overline{X_{k}}\right\|^{2}\right)\right)^{\frac{1}{1-m}}}{\sum_{t=1}^{c}\left(\sum_{k=1}^{s} \omega_{k}^{\alpha}\left(\left\|x_{j k}-a_{t k}\right\|^{2}-\eta_{t}\left\|a_{t k}-\overline{X_{k}}\right\|^{2}\right)\right)^{\frac{1}{1-m}}}
\end{gathered}
$$

\subsection{Discussions on WFCS}

In those equations above, $\eta_{i}\left\|a_{i}-\bar{X}\right\|^{2}$ decides the crisp kernel size of $i$ th cluster (2-dimensional diagram is shown in Figure 1) and $\eta_{i}$ guarantee that no two crisp kernels will overlapped [14]. We note that if data points fall on the crisp boundary (see Figure 1), the membership value $\mu_{i j} \rightarrow+\infty$ and the feature weighting $\omega_{k}$ will be negative, which are unacceptable. So, the membership value and feature weighting should be adjusted.

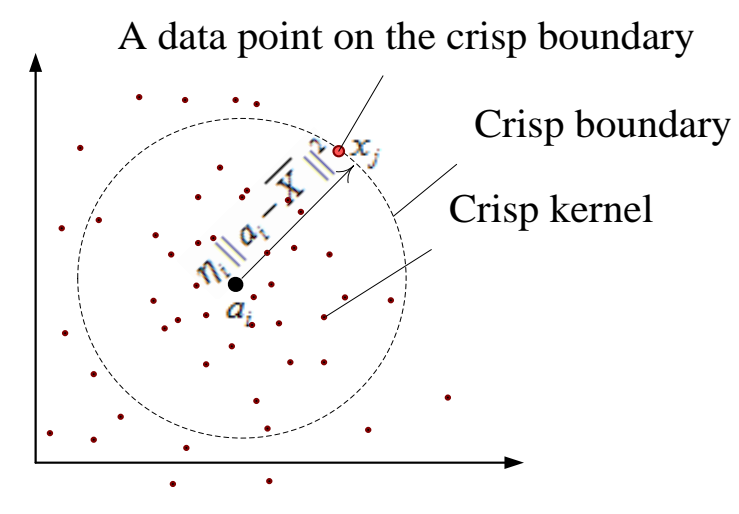

Figure 1. Illustration of the Crisp Kernel

(1) Adjustment of $\omega_{k}$

By denoting $\Delta_{k}=\sum_{i=1}^{c} \sum_{j=1}^{n} \mu_{i j}^{m}\left(\left\|x_{j k}-a_{i k}\right\|^{2}-\eta_{i}\left\|a_{i k}-\overline{X_{k}}\right\|^{2}\right)$, Equation (8) can be written as below:

$$
\omega_{k}=\frac{\Delta_{k}^{\frac{1}{1-\alpha}}}{\sum_{t=1}^{s} \Delta_{k}^{\frac{1}{1-\alpha}}}
$$


If $\Delta_{k}<0, \Delta_{k}$ will be projected to the positive space because $\omega_{k}$ must be nonnegative. We can adjust the feature weighting as follow:

$$
\omega_{k}=\left\{\begin{array}{cc}
0, & \Delta_{k}=0 \\
\frac{\Delta_{k}^{\frac{1}{1-\alpha}}}{\sum_{t=1}^{s} \Delta_{t}^{\frac{1}{1-\alpha}}}, & \Delta_{k}>0 \\
\frac{\Delta_{k}^{\frac{1}{1-\alpha}}}{\sum_{t=1}^{s} \Delta_{t}^{\frac{1}{1-\alpha}}}, & \Delta_{k}<0
\end{array}\right.
$$

where $\Delta_{k}^{\prime}=\Delta_{k}-\min \left(\Delta_{p}\right)+\min \left(\Delta_{q}\right), \Delta_{p}$ and $\Delta_{q}$ denote those are smaller than 0 and larger than 0 , respectively.

(2) Adjustment of $\mu_{i j}$

By denoting $\Delta_{i j}=\sum_{k=1}^{s} \omega_{k}^{\alpha}\left(\left\|x_{j k}-a_{i k}\right\|^{2}-\eta_{i}\left\|a_{i k}-\overline{X_{k}}\right\|^{2}\right)$, Equation (9) can be presented as following:

$$
\mu_{i j}=\frac{\left(\Delta_{i j}\right)^{\frac{1}{1-m}}}{\sum_{i=1}^{c}\left(\Delta_{i j}\right)^{\frac{1}{1-m}}}
$$

If $\Delta_{i p}$ equals to zero, it means that a data point $x_{p}$ falls on the $i$ th crisp boundary. Furthermore, the membership values of $x_{p}$ is fuzzier than that of data points in the crisp kernel and is greater than that of data points lying outside the crisp kernel. For those data points lying outside the crisp boundary, the $\Delta_{i t}$ is greater than zero. We denote $\Delta=\Delta_{i t} \cup \Delta_{i p}$, and $\Delta$ should be projected by Equation(13) before membership values be calculated by Equation(9).

$$
\Delta^{\prime}=\Delta+\min \left(\Delta_{i t}\right)
$$

\section{Fault Diagnosis using WFCS}

In this section, we detailed the approach for fault diagnosis using WFCS. Both simulated data and real data are conducted on WFCS and results are compared with the un-weighted clustering algorithm (FCS) and the feature weighting fuzzy clustering algorithm (WFCM). We choose a CFM56 type engines fault dataset with measurement noise as real data, and simulated engine gas path degradation data with Gauss noise (be denoted by GasPath) as simulated data (shown in Table 1). CFM56 type engine dataset consists of 3 classical fault types which are blade damage, oil leaking and bearing damage; simulated degradation datasets consist of FAN, HPC and LPT components degradation data in takeoff and cruise scenarios respectively. 
Table 1. Summary of Datasets Information

\begin{tabular}{cclc}
\hline Dataset & Scenario & \multicolumn{1}{c}{ Codes(fault) } & Numbers of dimension \\
\hline \multirow{3}{*}{ CFM56 } & cruise & F1(blade damage) & 3 \\
& & F2(oil leaking) & \\
& & F3(bearing damage) & \\
& F4-1(FAN degradation) & \\
cruise & F5-1(HPC degradation) & 15 \\
& & F6-1(LPT degradation) & \\
& \multirow{3}{*}{ takeoff } & F4-2(FAN degradation) & \\
& & F5-2(HPC degradation) & \\
& F6-2(LPT degradation) & \\
\hline
\end{tabular}

\subsection{Steps of Aero-Engine Fault Diagnosis Using WFCS}

Step 1. Collect and preprocess the multi-sensor data $X$;

Step 2. Set number of fault type $c$, parameter $\beta$, fuzzier exponent $m$, weighting exponent $\alpha$ and the iterative error threshold $\varepsilon$. Assign a random membership partition matrix $\left\{\mu_{i j}\right\}$ and random values between 0 and 1 to $\eta$. Set the initial iteration variant $l=1$;

Step 3. Update $a_{i}^{(l)}$ with $\mu_{i j}^{(l-1)} 、 \eta_{i}^{(l-1)}$ using Equation (7);

Step 4. Update and adjust $\omega_{k}^{(l)}$ with $\mu_{i j}^{(l-1)} 、 a_{i}^{(l-1)} 、 \eta_{i}^{(l-1)}$ using Equation (8) and Equation (11);

Step 5. Update and adjust $\mu_{i j}^{(l)}$ with $\omega_{k}^{(l-1)} 、 a_{i}^{(l-1)} 、 \eta_{i}^{(l-1)}$ using Equation (9) and Equation (13);

Step 6. Compute $\eta_{i}^{(l)}$ with $\beta$ 、 $a_{i}^{(l)}$ using Equation (4);

Step 7. $l=l+1$ and return to Step 2 , until convergence has been reached;

Step 8. Determine the fault type according to $\max _{i} \mu_{i j}$.

\subsection{Experiment Results and Discussion}

We evaluate the proposed method performance through the average recognition number (avg_RN). The avg_RN is defined as : avg _ $R N=\frac{1}{\text { trials }} \sum_{i=1}^{\text {trials }}\left(n_{\text {correct }}\right)_{i}$, where $n_{\text {correct }}$ means the number of what had been recognized correctly. The parameters $m$ and $\alpha$ can be determined based on the best average result. We set the fuzzier exponent $m=2$ and the threshold $\varepsilon=10^{-6}$, and choose $\alpha=2$ for CFM56 type engine dataset. For simulated dataset, we set $\alpha=-1$ and $\alpha=-10$ in cruise scenario and takeoff scenario respectively. The parameter $\beta$ involved in FCS and WFCS can be set as 0.005 . We conduct each algorithm for trials $=30$ on the three datasets.

The clustering results of three algorithms (FCS, WFCM and WFCS) are presented in Table 2. 
Table 2. The Results of the Three Algorithms

\begin{tabular}{clcccc}
\hline \multirow{2}{*}{ Dataset } & \multirow{2}{*}{ Codes } & \multicolumn{3}{c}{ avg_RN } & $\begin{array}{c}\text { Numbers of } \\
\text { fault instances }\end{array}$ \\
\cline { 3 - 5 } CFM56 & F1 & 7 & WFCM & WFCS & 18 \\
& F2 & 3 & 3 & 4 & 6 \\
& F3 & 5 & 6 & 10 & 13 \\
& F4-1 & 26 & 26 & 26 & 26 \\
& F5-1 & 14 & 14 & 14 & 14 \\
Gas Path & F6-1 & 9 & 13 & 15 & 23 \\
& F4-2 & 15 & 28 & 29 & 29 \\
& F5-2 & 16 & 16 & 28 & 28 \\
& F6-2 & 6 & 19 & 19 & 26 \\
\hline
\end{tabular}

Table 2 shows that F1, F2 and F3 can be recognized well by WFCS, and WFCM, FCS orderly. It shows that the WFCS can work well in engineering application.

As simulated data dimension increasing, we can see that weighting clustering algorithms performant better than un-weighting clustering algorithm, and all three algorithms get better results than what they get in real low-dimension dataset. WFCS, FCS and WFCM can detect all F4-1 and F5-1 correctly. WFCS can also classify F4-2 and F5-2 very well. For simulated datasets, we collected 15 sensors data for fault diagnosis, for example NL, NH, P13, P3, T3 and T6 etc. Different gas path components of aeroengine degradation may show the similar trend or value of data, which is difficult for clustering. So some instances of F6-1 are misclassified into F5-1 and some instances of F6-2 are misclassified into F5-2. Because that the number of fault instances in takeoff scenario are more than that of in takeoff scenario, so do the outliers. For simulated datasets, we can see that the average recognition rate of FCS and WFCM are declined for F4-2, F5-2 and F6-2, but the average recognition rate of WFCS for F4-2 and F5-2 is still $100 \%$ and get larger in F6-2.

From the final results, WFCS has the highest performance which shows WFCS is robust and competitive with the other two algorithms in fault diagnosis.

\section{Conclusions and Future Works}

In this paper, we introduced features weighting into FCS, discussed on WFCS and proposed a new method for aero-engine fault diagnosis with multi-sensor data by using WFCS. Experimental results on three datasets show that WFCS outperforms classical clustering algorithms (such as FCS and WFCM) in aero-engine fault diagnosis, which can handle high dimensional data well and doesn't need large number of fault instances.

The nature of proposed method is implemented on linear fault data. What if a highdimensional nonlinear fault data? For example, some aero-engines are based on nonlinear model and the collected sensor data are nonlinear. Common methods are to project data into a linear space by kernel function [15-17], we are very interested in incorporating our idea into the classical methods. It is a challenging problem and we put this endeavor for future work.

\section{Acknowledgments}

This work is supported by National Natural Science Foundation of China (Grant No.61403198), National Natural Science Foundation of China (Grant No.61501245). 


\section{References}

[1] P. Baraldi, R. Razavi-Far and E. Zio, "Classifier-ensemble incremental-learning procedure for nuclear transient identification at different operational conditions", Reliability Engineering and System Safety, vol. 98, no. 4, (2011), pp. 480-488.

[2] V. Macian, B. Tormos, P. Olmeda and L. Montoro, "Analytical approach to wear rate determination for internal combustion engine condition monitoring based on oil analysis", Tribology International, vol. 36, no. 10, (2003), pp. 771-776.

[3] T. Breikin, G. Kulikov, V. Arkov and P. Dynamic, "Modelling for Condition Monitoring of Gas Turbines: Genetic Algorithms Approach", in 16th IFAC World Congress, (2005).

[4] G. Niu, B. Yang and M. Pecht, "Development of an optimized condition-based maintenance system by data fusion and reliability-centered maintenance”, Reliability Engineering and System Safety, vol. 95, no. 7, (2010), pp. 786-796.

[5] P. Tamilselvan and P. Wang, "Failure diagnosis using deep belief learning based health state classification", Reliability Engineering \& System Safety, vol. 115, (2013), pp. 124-135.

[6] J. Hartigan, Clustering Algorithms. Wiley, New York, (1975).

[7] A.K. Jain, M.N. Murthy and P.J. Flynn, "Data clustering: a review", ACM Comput.Surv., vol. 31, no. 3, (1999), pp. 264-323.

[8] L.A. Zadeh, "Fuzzy sets", Inform. Contr., vol. 8, (1965), pp. 338-353.

[9] J.C. Dunn, "A fuzzy relative of the ISODATA process and its use in detecting compact well-separated clusters", J. Cybernet., no. 3, (1973), pp. 32-57.

[10] J.C. Bezdek, "Pattern Recognition with Fuzzy Objective Function Algorithm”, Plenum Press, New York, (1981).

[11] N. Zahid, M. Limouri and A. Essaid, "A new cluster-validity for fuzzy clustering”, Pattern Recognition, vol. 32, (1999), pp. 1089-1097.

[12] J. Z. Huang, M. K. Ng, H. Rong and Z. Li, "Automated variable weighting in k-means type clustering", Pattern Analysis and Machine Intelligence, IEEE Transactions on, vol. 27, no. 5, (2005), pp. 657-668.

[13] Q. Wang, Y. Ye and J.Z. Huang, "Fuzzy k-means with variable weighting in high dimensional data analysis", Web-Age Information Management, 2008. WAIM'08. The Ninth International Conference on. IEEE, (2008), pp. 365-372.

[14] K. L. Wu, J. Yu and M S. Yang, "A novel fuzzy clustering algorithm based on a fuzzy scatter matrix with optimality tests”, Pattern Recognition Letters, vol. 26, no. 5, (2005), pp. 639-652.

[15] J. M. Lee, C. K. Yoo, S. W. Choi and P.A. Vanrolleghem, "Nonlinear process monitoring using kernel principal component analysis", Chemical Engineering Science, vol. 59, no. 1, (2004), pp. 223-234.

[16] V. Roth and V. Steinhage, "Nonlinear discriminant analysis using kernel functions", Advances in neural information processing systems, (1999).

[17] B. Scholkopft and K. R. Mullert, "Fisher discriminant analysis with kernels", Neural networks for signal processing IX, (1999).

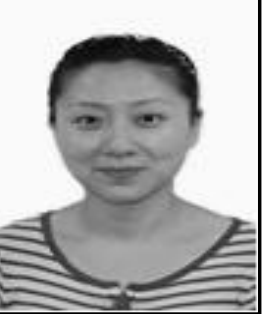

Yuan Zhou, she received the B.S. degree in system analysis and integration from Nanjing University of Information Science and Technology, China, in 2006, the M.S. degrees in computer science from Nanjing University of Information Science and Technology, China, in 2001. Now she is a PhD in Nanjing University of Aeronautics and Astronautics, China. Her main research interests include PHM of aero-engine, pattern recognition. 
International Journal of Control and Automation

Vol. 10, No. 5 (2017) 